\title{
EDITORIAL
}

\section{The right atrium as an anatomic set-up for re-entry: electrophysiology goes back to anatomy}

\section{F G Cosío}

Rapid, complete, uniform activation of all atrial tissue is important for rhythm stability. Typical flutter, the most common among atrial tachycardias, has anisotropic conduction at the terminal crest as an essential part of its mechanism

Correspondence to: Dr Francisco G Cosío, Cardiology Service, Hospital Universitario de Getafe, Carretera de Toledo, km 12.5, 28905 Getafe, Madrid, Spain; fcosio@vitanet.nu
N ormal heart activation is characterised by regular alternation between depolarisation/repolarisation and rest. Activation originates in the right atrium, around the sinus node area, and spreads until the atria are completely activated. Once activation is complete atrial tissue is refractory and a period of rest necessarily precedes the next activation cycle. This sequence is caused by pacemaker cells with discharge rates that respond to neural and humoral stimuli, thus allowing adaptation of heart function to physiologic demands. However, this normal sequence is not so simple to sustain, as demonstrated by the frequent appearance of re-entrant tachycardias.

Rapid, complete, uniform activation of all atrial tissue is important for rhythm stability. Preferential conduction pathways have been long recognised in the atria, ${ }^{1-3}$ despite the absence of bundles of specialised conduction akin to the His-Purkinje network of the ventricles. ${ }^{4}$ James and Sherf ${ }^{5}$ attributed the faster conduction along the terminal crest and Bachmann's bundle to the presence of specialised cells; however, present day thinking explains this by the anisotropic properties of atrial myocardium.

\section{ANISOTROPY}

In cardiac electrophysiology "anisotropy" refers to changes in conduction dependent on the anatomic orientation of myocardial fibres. According to the cable theory, conduction velocity depends on myocardial action potential upstroke-that is, the velocity of intracellular voltage change from negative to positive at the time of depolarisation. ${ }^{6}$ A decrease in the rate of depolarisation would cause conduction slowing as, for example, when antiarrhythmic drugs block the fast sodium $\left(\mathrm{Na}^{+}\right)$ channel. But a new approach was developed in the early 1980s when Spach showed that in atrial myocardial bundles packed longitudinally (pectinate muscle fragments) conduction could be faster along the main axis of the bundle than across it, despite a slower rate of action potential upstroke. ${ }^{7}$ The importance of intercellular coupling in determining conduction then began to be understood. Low resistance intercellular end-toend connections are responsible for faster con- duction along the longitudinal axis of the fibres, with respect to conduction across this axis. This effect can be most significant in complex structures where fibres change directions at sharp angles or cross in different directions. ${ }^{8}$ The effect of fibre "packing" is enhanced by local differences in gap junction distribution. Saffitz and colleagues ${ }^{9}$ showed that in ventricular myocardium, gap junction distribution is fairly even end-to-end and side-to-side. In this setting anisotropy is relatively modest, longitudinal conduction being three times as fast as transverse conduction. On the other hand, in the terminal crest gap junctions are almost exclusively disposed end-to-end and conduction velocity is 10 times faster in the longitudinal than in the transverse direction.

A normal, tightly packed, well organised atrial muscle structure can have an organising role for atrial activation, by helping rapid and uniform impulse conduction, thus preventing re-entrant activation. While Maze surgery directs activation linearly by creating barriers, ${ }^{10}$ normal anisotropy could have the same effect by speeding up conduction along the preferred anatomic axis. But anisotropy also has its traps. Spach extended his observations to a number of specific anatomic singularities, such as bifurcations, crossings, and perpendicular bundle insertions, and he could show that anisotropy could account for slow conduction, unidirectional block, and re-entry. ${ }^{78}$ And re-entry is the most frequent mechanism of tachycardias.

\section{ATRIAL FLUTTER}

Typical flutter, the most common among atrial tachycardias, has anisotropic conduction at the terminal crest as an essential part of its mechanism. ${ }^{11-13}$ Re-entry is a simple concept, but not an easy one to picture in the real anatomy of the heart. A ring of tissue, such as Mines made by cutting the heart of a tortoise, ${ }^{14}$ is ideal for continuous circular activation, because there is no way for activation to turn back on itself or cut across to the opposite side of the ring, disturbing the process. Although such a ring does not exist in the normal heart, a more-or-less circular band of myocardium can be formed by appropriately placed obstacles. In 1947 Rosenblueth and García-Ramos ${ }^{15}$ created such a band in the right atrium of dogs by crushing the myocardium between the superior and inferior caval vein orifices (fig 1). In this preparation activation could rotate permanently between an anterior border, made by the tricuspid ring, and a posterior border made by the crushing injury and the openings of both caval veins. 


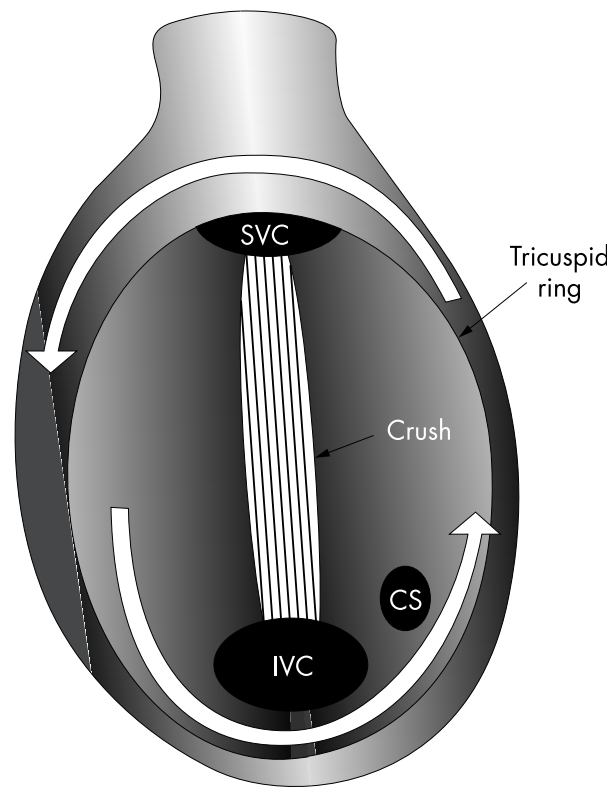

Rosenblueth's dog model

When Puech described the typical flutter circuit $^{16}$ after his endocardial mapping data, he basically reproduced Rosemblueth's circuit, activation rotating around the tricuspid ring. However, no insight was offered on a posterior obstacle preventing short circuiting or turning back of activation. It is more recently that we and others ${ }^{11}{ }^{12}$ have been able to determine that in human typical flutter there is indeed a posterior obstacle, albeit a functional one, based on anisotropy at and around the terminal crest. Endocardial recordings obtained with catheters, precisely placed over the crest with echocardiographic guidance, reveal double potentials, reflecting separate activation on both sides of the functional line of block. Thus the terminal crest combines with the orifices of the superior vena cava (SVC) and inferior vena cava (IVC) to make the large posterior obstacle that binds the circuit posteriorly, just as with Rosemblueth's crushing injury (fig 1).

But the role of anisotropy in the flutter circuit may not end just here. Relatively slow conduction has been described in the isthmus between the anterior rim of the IVC and the inferior rim of the tricuspid ring (IVC-TV isthmus), ${ }^{17}$ and this may be important both for initiation and maintenance of re-entry. Fine studies of fibre orientation in the isthmus ${ }^{18}{ }^{19}$ show terminal ramifications of the crest running perpendicular to insert in the tricuspid ring, thus making again a perfect anatomic set-up for local conduction slowing during circular activation (fig 1).

The study by Sánchez Quintana and colleagues ${ }^{20}$ in this issue of Heart is a unique systematic description of the muscular and fibrous components of this structure in the human that will greatly help us understand the relations between anatomy and electrophysiology in this critical area. Particularly interesting is the complex relation between the tightly packed, parallel myocardium of the crest itself and the fibres making the pectinate muscles, connected to the crest in varying angles that should favour block during rapid activation. It has been proposed that in human flutter transverse block may occur close to, but not precisely at, the terminal crest level, ${ }^{21}$ and recent experimental work showed how block can occur preferentially at the junction of the terminal crest with the pectinate muscles region. ${ }^{22}$

Sánchez Quintana and colleagues lament not having been able to study hearts which have borne atrial flutter throughout life. This is an important point, because with very few exceptions all human hearts have a tricuspid ring, an SVC, an IVC, and a terminal crest, but only very few develop flutter
Figure 1 Schematic representation of posterior right atrial wall barriers making macroreentry possible. On the left the crush injury made by Rosenblueth and García-Ramos ${ }^{15}$ in their dog experimental model allows circular activation around the tricuspid ring. On the right the position of the human terminal crest is represented. Note that its longitudinal axis is a barrier to transverse conduction, akin to Rosemblueth's crush injury. Terminal branching of the crest at the inferior vena cava-tricuspid ring isthmus may also facilitate re-entry by slowing conduction locally (wavey arrow) IVC, inferior vena cava; SVC, superior vena cava.

in their whole lives. Amazingly, very few data are available on the anatomy of the right atrium in flutter, including the presence and degree of dilatation and/or hypertrophy. Only preliminary intracardiac echocardiographic data ${ }^{23}$ suggest that terminal crest thickness can be larger in patients with atrial flutter than in those with fibrillation, and this would fit nicely with the higher transverse blocking capability of the crest in patients with atrial flutter ${ }^{24}$ compared with those with fibrillation. Fortunately atrial flutter is a relatively benign arrhythmia so that we will have to await a wide collaborative effort to obtain data on its anatomic bases, especially in relation with its more frequent relative, atrial fibrillation.

\section{REFERENCES}

1 Durrer D, van Dam RT, Freud GE, et al. Total excitation of the isolated human heart. Circulation 1970;41:899-12.

2 Waldo AL, Bush HL, Gelband H, et al. Effects on the canine P wave of discrete lesions in the specialized atrial tracts. Circ Res 1971;29:452-67

3 Boineau JP, Canavan TE, Schuessler RB, et al. Demonstration of a widely distributed atrial pacemaker complex in the human heart. Circulation 1988:77:1221-37.

4 Wand K, Ho SY, Anderson RH. Architecture of atrial musculature in humans. Br Heart J 1995;73:559-65.

5 Sherf $L$, James TN. Fine structure of cells and their histologic organization within internodal pathways of the heart: clinical and electrocardiographic implications. Am J Cardiol 1979;44:345-69.

6 Kléber AG, Janse M, Fast VG. Normal and abnormal conduction in the heart. In: Handbook of physiology. Section 2. The cardiovascular system. Volume 1: the heart. Oxford: Oxford University Press, 2001:455-530.

7 Spach MS, Miller WT, Geselowitz DB, et al. The discontinuous nature of propagation in normal canine cardiac muscle. Evidence for recurrent discontinuities of intracellular resistance that affect the membrane currents. Circ Res 1981;48:39-54

8 Spach MS, Miller WT, Dolber PC, et al. The functional role of structural complexities in the propagation of depolarization in the atrium of the dog: cardiac conduction disturbances due to discontinuities of effective atrial resistivity. Circ Res 1982;50:175-91.

9 Saffitz JE, Kanter L, Green KG, et al. Tissue-specific determinants of anisotropic conduction velocity in canine atrial and ventricular myocardium. Circ Res 1994:74:1065-70.

10 Cox JL, Canavan TE, Schuessler, et al. The surgical treatment of atrial fibrillation. II: intraoperative electrophysiologic mapping and description of the electophysiologic basis of atrial flutter and atrial fibrillation. J Thorac Cardiovasc Surg 1991;101:406-26.

11 Cosío FG, Arribas F, Barbero JM, et al. Validation of double spike electrograms as markers of conduction delay or block in atrial flutter. Am J Cardiol 1988;61:775-80

12 Olgin JE, Kalman JM, Fitzpatrick AP, et al. Role of right atrial structures as barriers to conduction during human type I atrial flutter. Activation and entrainment mapping guided by intracardiac echocardiography. Circulation 1995;92:1839-48. 
13 Saoudi N, Cosio F, Waldo A, et al. Classification of atrial flutter and regular atrial tachycardia according to electrophysiologic mechanism and anatomic bases: a statement from a joint expert group from the working group of arrhythmias of the European Society of Cardiology and the North Amerian Society of Pacing and Electrophysiology. Eur Heart J 2001;22:1162-82.

14 Mines GR. On dynamic equilibrium in the heart. J Physiol 1913;46:349-83.

15 Rosenblueth A, García Ramos J. Estudios sobre el flútter y la fibrilación. II. La influencia de los obstáculos artificiales en el flútter auricular experimental. Arch Inst Cardiol Mex 1947:17:1-19.

16 Puech P, Latour H, Grolleau R. Le flutter et ses limites. Arch Mal Coeur 1970;63:1 16-44.

17 Tai C-T, Chen S-A, Chiang C-E, et al. Characterization of low right atrial isthmus as the slow conduction zone and pharmacological target in typical atrial flutter. Circulation 1997:96:2601-11.

18 Cabrera JA, Sanchez-Quintana D, Ho SY, et al. The architecture of the atrial musculature between the orifice of the inferior caval vein and the tricuspid valve: the anatomy of the isthmus. J Cardiovasc Electrophysiol 1998;14:1186-95.
19 Waki K, Saito T, Becker AE. Right atrial flutter isthmus revisited: normal anatomy favors nonuniform anisotropic conduction. Circulation 1999;99:3017-23.

20 Sánchez Quintana D, Anderson RH, Cabrera JA, et al. The terminal crest: morphological features relevant to electrophysiology. Heart 2002;88:406-11.

21 Friedman PA, Luria D, Fenton AM, et al. Global right atrial mapping of human atrial flutter: the presence of posteromedial (sinus venosa region) functional block and double potentials: a study in biplane fluoroscopy and intracardiac echocardiography. Circulation 2000;101:1568-77.

22 Becker R, Baver A, Metz S, et al. Intercaval block in normal canine hearts: role of the terminal crest. Circulation 2001;103:2521-6.

23 Suzuki A, Kuga K, Enomoto M et al. Findings of intracardiac echocardiography of the crista terminalis in patients with atrial flutter [abstract]. Circulation 2001;102:11-619.

24 Shumacher B, Jung W, Schmidt $\mathrm{H}$, et al. Transverse conduction capabilities of the crista terminalis in patients with atrial flutter and atrial fibrillation. J Am Coll Cardiol 1999;34:363-73.

\section{IMAGES IN CARDIOLOGY}

\section{Right coronary artery aneurysm diagnosed with multislice computed tomographic angiography}

A

n 83 year old man presented with shortness of breath. Physical examination revealed a raised jugular venous pressure and atrial fibrillation with a rapid ventricular response. A provisional diagnosis of congestive cardiac failure was made and treatment with digoxin and diuretics initiated. A transthoracic echocardiogram demonstrated a large "doughnut" shaped extracardiac mass compressing and displacing the right atrium.

Non-invasive coronary angiography was performed using multislice helical ("spiral") computed tomography (CT). The breath hold required for a scan time of 31 seconds was well tolerated. Following contrast injection images were acquired using a gantry rotation time of $500 \mathrm{~ms}$ and retrospective electrocardiographic gating. Images were then reconstructed using the diastolic phase. Despite the lack of a regular R-R interval, excellent image resolution was obtained.

Axial transverse reconstruction (multiplanar reconstruction or MPR) CT coronary angiography (upper panel) demonstrated a right coronary artery aneurysm. The right coronary artery is shown arising from the aorta (Ao). The contrast filled lumen (An) of the aneurysmal portion of the artery is surrounded by unenhanced thrombus, which explained the echocardiographic appearances. The aneurysm is compressing the right atrium. A three dimensional reconstruction (using volume rendering techniques or VRT) shows the lumen of the aneurysm (An in lower panel) without the surrounding unenhanced mural thrombus. Although this type of "visual" reconstruction provides excellent information for surgeons, the treatment in this case was medical in view of the patient's frailty and his good response to rate controlling drugs. C Roobottom A J Marshall hughesgi@talk21.com
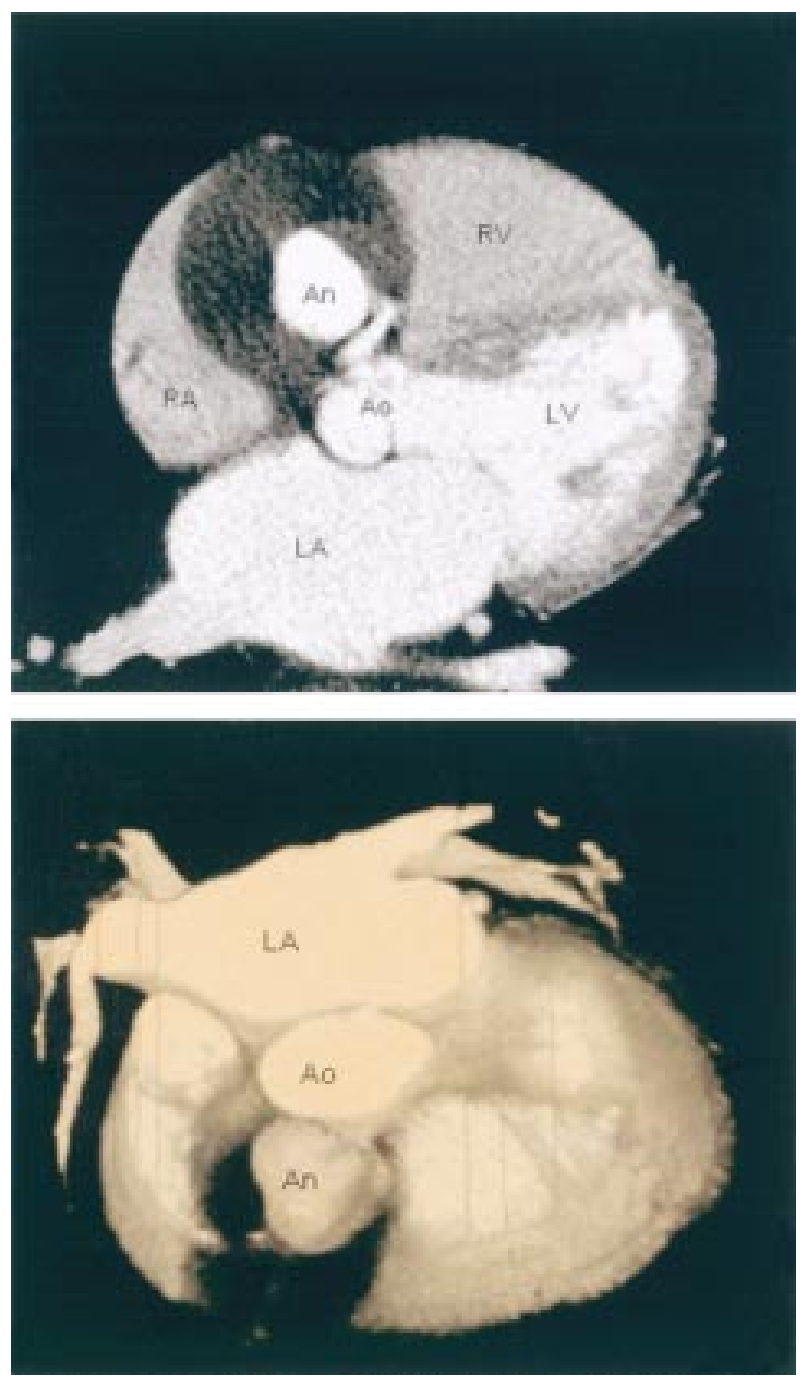